\title{
Multi-GeV lightcurves: possible hints for the emission mechanism
}

\author{
Katsuaki Asano* \\ Interactive Research Center of Science, Graduate School of Science, \\ Tokyo Institute of Technology, \\ 2-12-1 Ookayama, Meguro-ku, Tokyo 152-8550, Japan \\ E-mail: asano@phys.titech.ac.jp

\section{Peter Mészáros} \\ Department of Astronomy \& Astrophysics; Department of Physics; \\ Center for Particle \& Gravitational Astrophysics; \\ Pennsylvania State University, \\ University Park, PA 16802 \\ E-mail: nnpeastro.psu.edu
}

\begin{abstract}
Although the Fermi observatory has reported delayed onsets of $\mathrm{GeV}$ emissions, the photon statistics are not sufficient to distinguish the emission mechanisms. Future multi-GeV observations such as CTA may give us precise lightcurves that can be compared with lower energy bands. In this paper we show our time-dependent simulations for leptonic and hadronic models. Our results suggest that the multi-GeV lightcurves are powerful tool to probe the $\mathrm{GeV}$ emission mechanisms.
\end{abstract}

Gamma-Ray Bursts 2012 Conference-GRB2012,

May 07-11, 2012

Munich, Germany

${ }^{*}$ Speaker. 


\section{Introduction}

The Large Area Telescope (LAT) on board the Fermi Gamma-Ray Space Telescope revealed that some fraction of GRBs show delayed onset of GeV emission as seen in GRB 080916C [1]. Furthermore, GRB 090510 [2], GRB 090902B [3], and GRB 090926A [4] have an extra spectral component that dominates $\mathrm{GeV}$ energy range. While such behaviours in $\mathrm{GeV}$ emissions can constrain the emission mechanisms, the photon statistics in Fermi telescope are still insufficient to confirm the correlation between $\mathrm{GeV}$ and $\mathrm{MeV}$ lightcurves quantitatively. The typical photon counts in the Fermi-LAT observations is a few per pulse. We may need hundreds of $\mathrm{GeV}$ photons or more to compare the widths of pulses with $\mathrm{MeV}$ pulses. In order to obtain enough photons above $\mathrm{GeV}$, a much larger effective area is required. Therefore, air Cherenkov telescopes (ACT) such as Cherenkov Telescope Array (CTA) etc. can be powerful tools, if the low-energy threshold is lowered as low as $10-30 \mathrm{GeV}$. If an ACT fortunately detect a GRB similar to the Fermi-LAT GRBs, the expected gamma-ray lightcurve may be so precise that we can compare its variability with that for lower energy bands. This must determine the origin of $\mathrm{GeV}$ emissions; external or internal.

If we confirm that the $\mathrm{GeV}$ sources are common to the $\mathrm{MeV}$ emission region, the delayed onset of $\mathrm{GeV}$ emission will be focused on as an enigmatic signature again. This can be a hint to unravel the emission mechanism. In this paper, we consider methods to distinguish several models that explain the delayed onset, from multi-GeV lightcurves observed with ACTs.

\section{SSC model}

The simple synchrotron self-Compton (SSC) model can naturally explain the extra components. In this model, the delayed onset can be interpreted as a lag-time before the seed photons for SSC build up. We, Asano \& Mészáros (2011) [5] (hereafter AM11) developed a time-dependent code to simulate emissions from a relativistically expanding shell. While our code is based on onezone approximation, it can follow the injection and cooling for electrons/positrons, and production, absorption and escape for photons. The physical processes we considered are (1) synchrotron (2) Thomson or inverse Compton (IC) scattering (including the Klein-Nishina regime) (3) synchrotron self-absorption (SSA) (4) $\gamma \gamma$ pair production (5) adiabatic cooling.

For moderete magenetic fields such as $\varepsilon_{B} / \varepsilon_{\mathrm{e}}=0.1-1$, it is hard to find a delayed onset comparable to the variability timescale $R / \Gamma^{2} / c$. Only if we adopt a very low magnetic field (due to small $\varepsilon_{B}$ such as $\varepsilon_{B} / \varepsilon_{\mathrm{e}} \sim 10^{-3}$ and/or large radius $R$ even for large $\varepsilon_{B}$ ), the delayed onset is reproduced by the slow growth of the seed photon field. In this case, the low magnetic field requires a large $\gamma_{\mathrm{e}, \mathrm{min}}$ $\left(>10^{4}\right)$ to make the spectral peak energy $\sim \mathrm{MeV}$, which implies that the Klein-Nishina effect is effective. This is a primary reason why the IC component grows slowly.

Only in this extreme parameter set, the one-zone leptonic model can reproduce a significant delayed onset. Even in this case, the width of the lightcurves for $\mathrm{MeV}$ and $\mathrm{GeV}$ is almost the same.

\section{EIC model}

Two-zone external inverse Compton (EIC) model was proposed by Toma et al. [6, 7] to explain the delayed onset of $\mathrm{GeV}$ emission. The $\mathrm{MeV}$ photons are emitted from a smaller radius, and 

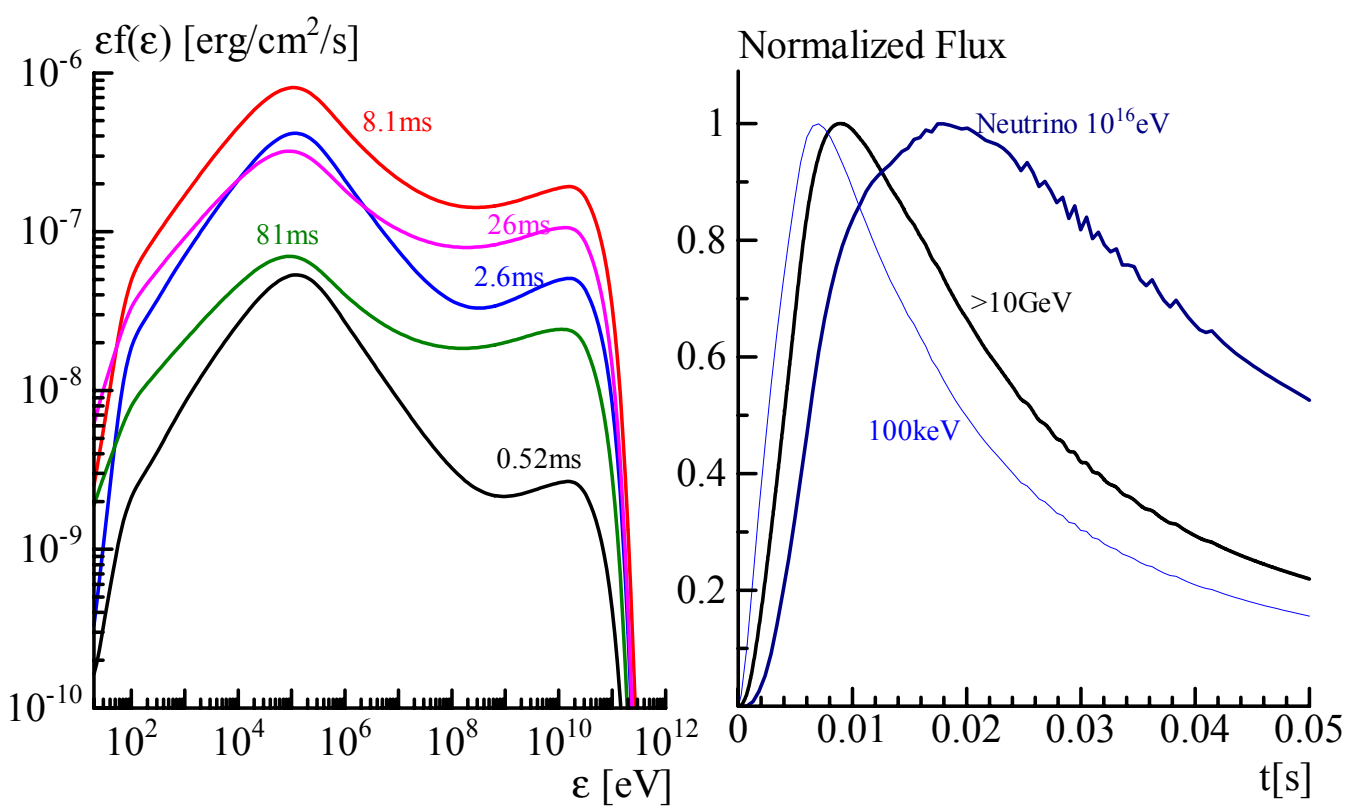

Figure 1: Spectral evolution (left) and lightcurves (right) for an observer $(z=1)$ in the hadoronic model. The model parameters are $\Gamma=800, R_{0}=10^{14} \mathrm{~cm}$, isotropic-equivalent energy of electrons $E_{\mathrm{e}, \mathrm{pls}}=5.0 \times 10^{50}$ erg, protons $E_{\mathrm{p}, \mathrm{pls}}=6.1 \times 10^{51} \mathrm{erg}$, and the initial magnetic field $B_{0}^{\prime}=1.9 \times 10^{4} \mathrm{G}$.

they are upscattered by accelerated electrons in an outer region. In this model, we require two different origins for the $\mathrm{MeV}$ and $\mathrm{GeV}$ components. A GeV delay larger than the typical pulse timescale appears naturally. In the frame of the outer dissipation region, the photon field coming from an inner region is highly beamed. As a result, the intensity of the scattered photons becomes anisotropic. In this case, the emission from off-axis regions $(\theta \gtrsim 1 / \Gamma)$ is not negligible. We also tested this case with our numerical code in AM11, and showed that the GeV lightcurve has a long tail, which is a characteristic feature of this model.

\section{Opacity evolution}

Using the same code in AM11, we also test the $\gamma \gamma$-opacity evolution effect [8]. At least in our one-zone formulation, an electron injection timescale longer than the expansion timescale is required in order to observe the opacity damping. As the opacity decreases with radius $R$, the cut-off energy increases. So in this model, the high-energy emissions tend to delay. However, the spectral shape at the cut-off energy is not so sharp even in one-zone approximation. The spectrum can be approximated by a broken power-law, which comes from the balance between photon production and absorption in the source. Thus, the model lightcurve shows gradual $\mathrm{GeV}$ onset, which seems different from the observed "sudden" onset. Thus, we conclude that the $\gamma \gamma$-opacity evolution is hard to explain the delay. 


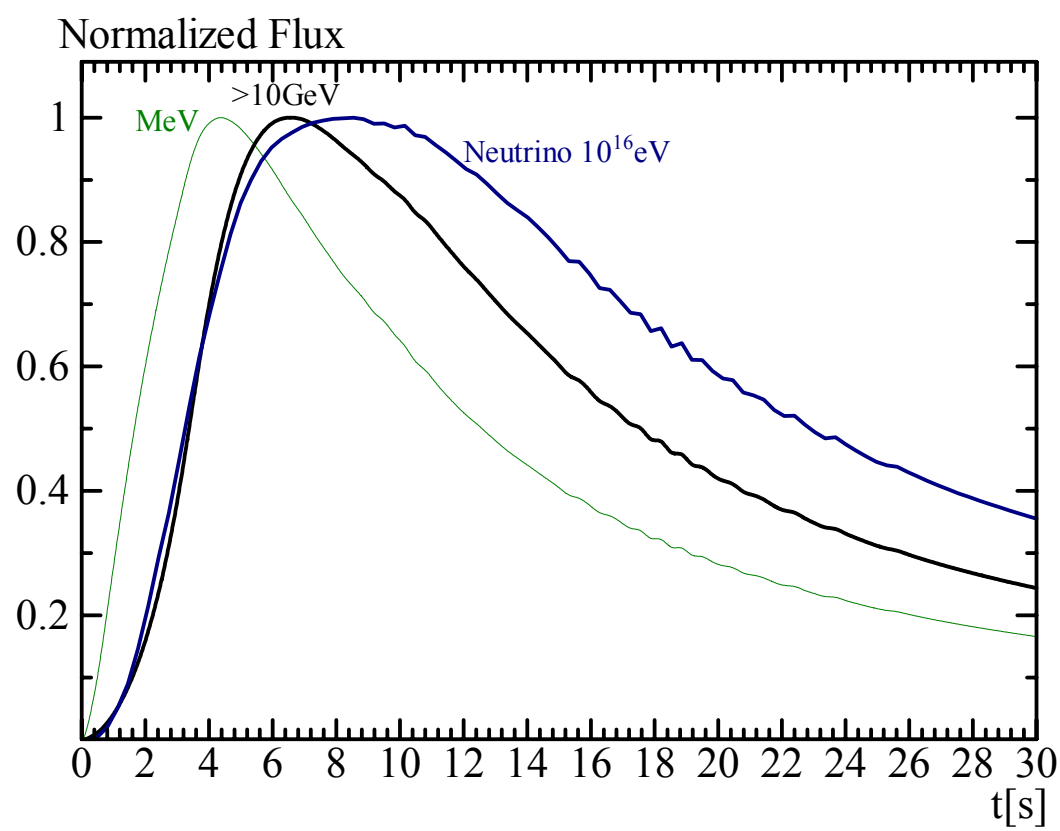

Figure 2: Lightcurves for an observer $(z=4.35)$ in the hadoronic model. The model parameters are $\Gamma=600$, $R_{0}=1.3 \times 10^{16} \mathrm{~cm}, E_{\mathrm{e}, \mathrm{pls}}=2.0 \times 10^{54} \mathrm{erg}, E_{\mathrm{p}, \mathrm{pls}}=4.8 \times 10^{55} \mathrm{erg}$, and $B_{0}^{\prime}=830 \mathrm{G}$.

\section{Hadronic model}

We installed hadronic processes into the code in AM11, and demonstrate the lightcurves for hadronic models [8]. Hadronic models can explain the extra spectral components by the sycnhrotron/IC emissions from electron-positron pairs produced via the electro-magnetic cascade triggered by photopion production (hadronic cascade) [9, 10]. The maximum energy of protons gradually increases during particle injection in the shocked shell. On the other hand, the injected electrons produce a soft photon field around $\mathrm{MeV}$. The spectrum of the $\mathrm{MeV}$ component is as soft as $\alpha \sim-1.5$, where $\alpha$ is the low-energy photon index. Therefore, the efficiency of photopion production grows with the proton energy. Therefore, until the maximum energy grows enough, the electro-magnetic cascade triggered by the photomeson production does not start. This yields the dalyed onset of $10 \mathrm{GeV}$ emissions relative to the $\mathrm{MeV}$ photon emission. Moreover, even after the particle injection is stopped, the residual photons in the source continuously interact with protons. As a result, the gamma-ray emissions due to the hadronic cascade last longer than the $\mathrm{MeV}$ emissions. An examples of the spectral evolution and lightcureves is shown in Figure 1. The characteristic feature is that the $10 \mathrm{GeV}$-lightcurve has a wider shape than the $\mathrm{MeV}$-lightcurve.

As shown in Figure 1, the neutrino lightcurve shows more distinctive delay than $10 \mathrm{GeV}$ delay. This is because the $\mathrm{GeV}$ photons are produced from not only hadronic processes but also IC emission from primary electrons.

If we adopt a large radius, even for a large $\varepsilon_{B}$, the magnetic field becomes so low that the Klein-Nishina effect becomes crucial. In this case, the IC emission is negligible in GeV band. As shown in Figure 2, the delays for $\mathrm{GeV}$ and neutrinos are comparable. 


\section{Conclusion}

The GeV delays can be explained either by a one-zone leptonic model with very low magnetic field, or by normal parameter range one-zone hadronic models; or also by a two-zone EIC leptonic model. However, the lightcurve behavior of these models differ substantially from each other. While the FWHMs of the $\mathrm{MeV}$ and $\mathrm{GeV}$ lightcurves are almost the same in one-zone leptonic models, the FWHM of the $1-30 \mathrm{GeV}$ lightcurves in hadronic models are significantly wider than those of the $0.1-1 \mathrm{MeV}$ lightcurves.

Here, we have assumed a simple Bohm-limit acceleration for electrons and protons. Of course, the lightcurve may depend on the model details of particle acceleration. The electron acceleration timescale is usually considered to be short enough compared to the dynamical timescale. However, the continuous acceleration like the second-order Fermi acceleration [11] or continuous heating [12] for electrons are recently focused on to resolve the contradictions in the low-energy spectral index etc. In such models, the lightcurve behaviour can be different from the examples shown here even in leptonic models. Another possible mechanism to delay $\mathrm{GeV}$ emission is the temporal evolution of the magnetic field [11]. The magnetic field amplified by some plasma instabilities can decay suddenly after a certain timescale provided by the plasma frequency. Then, IC emission becomes dominant instead of synchrotron emission. The predicted differences in the lightcurves for various models including the above models can be tested with future atmospheric Cherenkov telescopes such as CTA.

\section{References}

[1] Abdo, A. A. et al., Fermi Observations of High-Energy Gamma-Ray Emission from GRB 080916C, Science 323 (2009) 1688

[2] Ackermann, M. et al., Fermi Observations of GRB 090510: a Short-Hard Gamma-Ray Burst with an Additional, Hard Power-Law Component from $10 \mathrm{keV}$ to GeV Energies, ApJ 716 (2010) 1178

[3] Abdo, A. A. et al., Fermi Observations of GRB 090902B: A Distinct Spectral Component in the Prompt and Delayed Emission, ApJ 706 (2009) L138

[4] Ackermann, M. et al., Detection of a Spectral Break in the Extra Hard Component of GRB 090926A, ApJ 729 (2011) 114

[5] Asano, K. \& Mészáros, P., Spectral-Temporal Simulations of Internal Dissipation Models of Gamma-Ray Bursts, ApJ 739 (2011) 103

[6] Toma, K., Wu, X.-F. \& Mészáros, P., An Up-Scattered Cocoon Emission Model of Gamma-Ray Burst High-Energy Lags ApJ 707 (2009) 1404

[7] Toma, K., Wu, X.-F. \& Mészáros, P., Photosphere-internal shock model of gamma-ray bursts: case studies of Fermi/LAT bursts, MNRAS 415 (2011) 1663

[8] Asano, K. \& Mészáros, P., Spectral-Temporal Simulations of Internal Dissipation Models of Gamma-Ray Bursts, submitted to ApJ (2012) [arXiv: 1206.0347 ]

[9] Asano, K., Guiriec, S., \& Mészáros, P., Hadronic Models for the Extra Spectral Component in the Short GRB 090510, ApJ 705 (2009) L191 
[10] Asano, K., Inoue, S., \& Mészáros, P., Prompt X-ray and Optical Excess Emission due to Hadronic Cascades in Gamma-Ray Bursts, ApJ 725 (2010) L121

[11] Asano, K., \& Terasawa, T., Slow Heating Model of Gamma-Ray Burst: Photon Spectrum and Delayed Emission, ApJ 705 (2009) 1714

[12] Beloborodov, A. M., Collisional mechanism for gamma-ray burst mission, MNRAS 407 (2010) 1033 